\title{
SMR
}

\section{Expression of microRNA-210 in tissue and serum of renal carcinoma patients and its effect on renal carcinoma cell proliferation, apoptosis, and invasion}

\author{
T.Y. Liu' ${ }^{1}$, H. Zhang' ${ }^{2}$ S.M. Dü ${ }^{3}$ J. Li ${ }^{1}$ and X.H. Wen ${ }^{1}$ \\ 'Department of Urology, Zhumadian Central Hospital, Zhumadian, China \\ ${ }^{2}$ Department of Nursing, Huanghuai University, Zhumadian, China \\ ${ }^{3}$ Minor Operation Room, Zhumadian Central Hospital, Zhumadian, China \\ Corresponding author: T.Y. Liu \\ E-mail: taiyangliu@yeah.net \\ Genet. Mol. Res. 15 (1): gmr.15017746 \\ Received September 29, 2015 \\ Accepted December 4, 2015 \\ Published March 4, 2016 \\ DOI http://dx.doi.org/10.4238/gmr.15017746
}

ABSTRACT. This study investigated the expression of microRNA-210 $(m i R-210)$ in tissue and serum of renal carcinoma patients and its effect on renal carcinoma cell proliferation, apoptosis, and invasion. Thirtytwo renal carcinoma patients in our hospital were selected as the study group and 32 people receiving a physical examination were selected as the control group. miR-210 expression in the serum of renal carcinoma patients and in healthy subjects was quantified by real-time polymerase chain reaction. After miR-210 overexpression and inhibition in ACHN cells in human renal carcinoma, ACHN cell proliferation, apoptosis, and invasion were detected by CCK-8, flow cytometry, and a transwell invasion assay. The expression of miR-210 was significantly higher in renal carcinoma than in corresponding paracarcinoma tissues $(P<0.001)$. The expression of miR-210 was significantly higher in the serum of renal carcinoma than in the control group $(P<0.001)$. ACHN cell proliferation and invasion were significantly increased and apoptosis was significantly decreased $(P$ $<0.05$ ) when miR-210 was overexpressed. ACHN cell proliferation and 
invasion were significantly decreased and apoptosis was significantly increased $(P<0.05)$ when miR-210 was inhibited. In conclusion, miR-210 was highly expressed in tissues and serum of renal carcinoma patients. miR-210 could promote the proliferation and invasion of renal carcinoma cells and inhibit the apoptosis of renal cell carcinoma cells.

Key words: MicroRNA-210 (miR-210); Renal cell carcinoma; Proliferation; Apoptosis; Invasion

\section{INTRODUCTION}

Renal cell carcinoma (RCC, abbreviated as renal carcinoma) is a malignant tumor with a highgrade malignancy in urinary systems. The incidence of RCC ranks seventh in male malignant cancer and twelfth in female malignant cancer, accounting for about 2-3\% of total malignant cancers (Cohen and McGovern, 2005). RCC has a poor response to chemotherapy and radiotherapy and surgery is the only effective therapeutic schedule at present (Doh et al., 2006). Thus, early diagnoses are very necessary for patients. For patients without metastasis, the five-year survival rate can reach $90 \%$. However, once tumor metastasis occurs, the five-year survival rate is less than 10\% (Drucker, 2005). It is necessary to understand the molecular mechanism of the occurrence, development, and metastasis of RCC.

MicroRNAs (miRNAs) are highly conserved noncoding RNAs that are about 18-24 nucleotides long. They inhibit the expression of target genes via complementary pairing of sequence seeds in the 3' untranslated region (3' UTR). miRNAs were first discovered in nematodes. The extensive expression of miRNAs has since been discovered in many organisms such as plants and mammals. More and more studies have proven that miRNAs regulate many biological processes, including cell development, proliferation, differentiation, apoptosis, hematopoiesis, metabolism, and tumorigenesis (Kim, 2005; Du and Zamore, 2005).

miRNA-210 (miR-210) is an miRNA located in the intron of the AK123483 gene (Giannakakis et al., 2008). It is highly expressed in many tumors. Previous studies have indicated that miR-210 is expressed in the form of hypoxia-inducible factor 1 (HIF-1) and HIF-2 dependency or HIF independency. miR-210 regulates the processes of tumor cell cycle, cell migration, invasion, differentiation, and angiogenesis by acting on different target genes (Zhang et al., 2009; Tsuchiya et al., 2011; Cicchillitti et al., 2012; Alaiti et al., 2012). miR-210 was found to be highly expressed in renal carcinoma and involved in angiogenesis of renal carcinoma through deep sequencing, microRNA chips, and other techniques (Zhang et al., 2009; Nakada et al., 2011; Tsuchiya et al., 2011; Cicchillitti et al., 2012; Alaiti et al., 2012; McCormick et al., 2013; Takikawa et al., 2013; Samaan et al., 2015). However, so far there are few reports on the expression of miR-210 in renal carcinoma and its effect on renal carcinoma cell proliferation, apoptosis, and invasion.

In this study, the expression of miR-210 in renal carcinoma tissues and corresponding paracarcinoma tissues in renal carcinoma patients were compared against miR-210 expression levels in the serum of healthy subjects. We also further investigated the effects of miR-210 on renal carcinoma cell proliferation, apoptosis, and invasion.

\section{MATERIAL AND METHODS}

\section{Clinical data}

All tissues were derived from surgical specimens of renal carcinoma patients who came 
to our hospital from August 2014 to August 2015. There were 32 cases, including 25 males aged 51-75 years old. The average age was 62.32 years. The patients did not receive any intervention chemotherapy or chemotherapy treatment before the operation. All patients underwent renal carcinoma radical resection. The tissue specimens were confirmed as renal carcinoma by postoperative pathology. The materials of carcinoma tissue and paracarcinoma tissue were removed in the intraoperative $30 \mathrm{~min}$ in vitro, fixed with $10 \%$ formalin, embedded with paraffin, and sliced for reverse. The control group included 32 health people, including 22 males aged 50-73 years old. The average age was 61.27 years old. There were no significant differences in gender or age $(P>$ 0.05 ) between the two groups (Table 1). This study was conducted in accordance with the Declaration of Helsinki. This study was conducted with approval from the Ethics Committee of Zhumadian Central Hospital. Written informed consent was obtained from all participants.

Table 1. Expression of miR-210 in the renal carcinoma and control groups.

\begin{tabular}{l|c|c|c|c}
\hline Groups & $\mathrm{N}$ & Male/female & Average age (years) & Serum miR-210 \\
\hline Renal carcinoma group & 32 & $25 / 7$ & $62.32 \pm 6.17$ & $0.92 \pm 0.18$ \\
\hline Control group & 32 & $22 / 10$ & $61.27 \pm 7.88$ & $5.03 \pm 0.82$ \\
\hline$\chi^{2}$ or $t$ & & 0.721 & 0.593 & 27.694 \\
\hline $\mathrm{P}$ & & 0.396 & 0.555 & 0.000 \\
\hline
\end{tabular}

Chi-square test used for gender comparison, $t$-test used for other comparisons.

\section{Real-time polymerase chain reaction (RT-PCR)}

Renal carcinoma and corresponding paracarcinoma tissues were removed during the operation. In addition, peripheral venous blood $(5 \mathrm{~mL})$ was extracted from the renal carcinoma patients and healthy subjects, stood, and centrifuged. The serum was separated. Trizol (1 mL; Invitrogen, Carlsbad, CA, USA) was added to about $150 \mathrm{mg}$ tissue or $200 \mu \mathrm{L}$ serum. Total RNA was extracted after sufficient homogenization. The isolated RNA was dissolved in $25 \mu \mathrm{L}$ DEPC water. RNA was quantified and purity was detected using an ultraviolet spectrophotometer. The Promega Reverse Transcription kit was used for reverse transcription (RT) (Promega, Madison, WI, USA). Template RNA $(2 \mu \mathrm{g})$ was mixed with miRNA RT primers $(4 \mu \mathrm{L})$. The mixture was incubated in a $70^{\circ} \mathrm{C}$ water bath for $10 \mathrm{~min}$ and a $0^{\circ} \mathrm{C}$ ice bath for 2-3 min. At the same time, the reaction system was prepared by mixing $2.5 \mathrm{mM}$ dNTPs $(4 \mu \mathrm{L}), 5 \mathrm{X}$ RT Buffer $(10 \mu \mathrm{L}), 200 \mathrm{U} / \mathrm{mL}$ reverse transcriptase $(1 \mu \mathrm{L})$, and $40 \mathrm{U} / \mathrm{mL}$ RNase inhibitor $(1 \mu \mathrm{L})$. After the ice bath incubation, the template-primer mixture was added into the reaction system and DEPC water was added to a final volume of $50 \mu \mathrm{L}$. After incubation at $42^{\circ} \mathrm{C}$ for $90 \mathrm{~min}$ and $95^{\circ} \mathrm{C}$ for $5 \mathrm{~min}$, the reverse transcription was immediately terminated in a $0^{\circ} \mathrm{C}$ ice bath for $5 \mathrm{~min}$. The resulting cDNA was used to quantify the corresponding miRNA via RT-PCR.

The Bulge-Loop ${ }^{\mathrm{TM}}$ miRNA qRT-PCR Primer Set $(5 \mu \mathrm{M})$ was used for RT-PCR. The corresponding miRNART-PCR primers were dissolved in distilled water to a final concentration of $5 \mu \mathrm{M}$. The reaction system was established according to the manufacturer protocol. The miR-210 and U6 reference primer were synthesized by Ribobio Co. Ltd., Guangzhou, China. The U6 snRNA primer sequences are as follows: RT: 5'-CGCTTCACGAATTTGCGTGTCAT-3'; PCR-F: 5'-GCTTCGGCA GCACATATACTAAAAT-3'; PCR-R: 5'-CGCTTCACGAATTTGCGTGTCAT-3'. The miRNA mimic negative control and miR-210 mimic primers were purchased from Jima Co., Shanghai, China. These primer sequences are as follows: RT: CGTGGAGTCGGCAATTGCACTGGATACGACCAGT GTG-3'; PCR-F: 5'-AGCCCCTGCCCACCGCAC-3'; PCR-R: 5'-GTGCAGGGTCCGAGGT-3'. The miRNA inhibitor negative control and miR-210 inhibitor were purchased from Exiqon Co., Denmark. The primer sequences for the miRNA mimic negative control were as follows: 5'-UUCUCCGAAC 
GUGUCACGUTT-3'; miR-210 mimic: 5'-AGCCCCUGCCCACCGCACACUG-3'. The 7900 Type Real-Time PCR instrument (Applied Biosystems, Foster City, CA, USA) was used. The relative quantitative results were analyzed using $2^{-\Delta \Lambda C t}$ method.

\section{CCK-8 cell proliferation experiment}

The miRNA mimic and inhibitor were transfected in human renal carcinoma ACHN cells using LipofectamineTM 2000 (ThermoFisher, USA). The cells were inoculated on a 96-well plate with 1000 cells/well $48 \mathrm{~h}$ after transfection. Six complex wells were set for each group. Cell proliferation was detected according to the CCK-8 kit protocol (Nanjing Nuoweizan Company) after 1 , 2,3 , and 4 days of culture. The OD value was detected at a 450-nm wavelength by an automatic microplate reader (Rayto Automatic Enzyme Mark Instrument Microplate Reader RT-6000).

\section{Flow cytometry detection}

ACHN cells were inoculated on a 6-well plate at $1 \times 10^{6}$ cells/well, transfected for $24 \mathrm{~h}$, washed twice with PBS, and resuspended with 1X Binding Buffer. Fluorescein isothiocyanate-labeled annexin $\mathrm{V}(50 \mu \mathrm{L}$; BD Biosciences, USA) and propidium iodide $(50 \mu \mathrm{L})$ were added, gentle mixed, and stained at room temperature away from light for $15 \mathrm{~min}$. Apoptosis was detected by flow cytometry.

\section{Transwell invasion experiment}

RPMI-1640 medium containing $10 \%$ serum $(500 \mu \mathrm{L})$ was added to a 24 -well plate. The transwell chamber was arranged in the well. BD matrigel (60 $\mu$ L) and RPMI-1640 (1:8) were added in each transwell chamber and incubated for $3 \mathrm{~h}$ in the cell incubator. Cells were suspended to a final concentration of $5 \times 10^{4}$ cells $/ \mathrm{mL}$ in pure RPMI-1640 medium $48 \mathrm{~h}$ after transfection. A $200-\mu \mathrm{L}$ aliquot of the cell suspension was added in each chamber, or about $1 \times 10^{4}$ cells/well. Six complex wells were set for every group. The 24 -well plate was placed into the cell incubator. The chambers were removed after 6 and $12 \mathrm{~h}$ of culturing and then they were stained and fixed. Five fields of view were randomly selected under the microscope, photographed, and counted. The number of cells entering the chamber was counted to confirm the cell invasion.

\section{Statistical analysis}

All data were analyzed using the SPSS 19.0 software (SPSS Inc., Chicago, IL, USA). The statistical charts were drawn using the GraphPad Prism 4.0 software. Observational data mainly included the measurement data, which passed normality tests. Differences between the two groups were compared using a $t$-test. ANOVA for repeated measures was conducted for multiple time-point data. $\mathrm{P}<0.05$ indicated that the difference was statistically significance.

\section{RESULTS}

\section{Expression of miR-210 in renal carcinoma tissues and corresponding paracarci- noma tissues}

Renal carcinoma tissues $(\mathrm{N}=32)$ were compared against paracarcinoma tissues. The 
expression of miR-210 was significantly higher $(P<0.05)$ in renal carcinoma tissues than in paracarcinoma tissues (Figure 1).

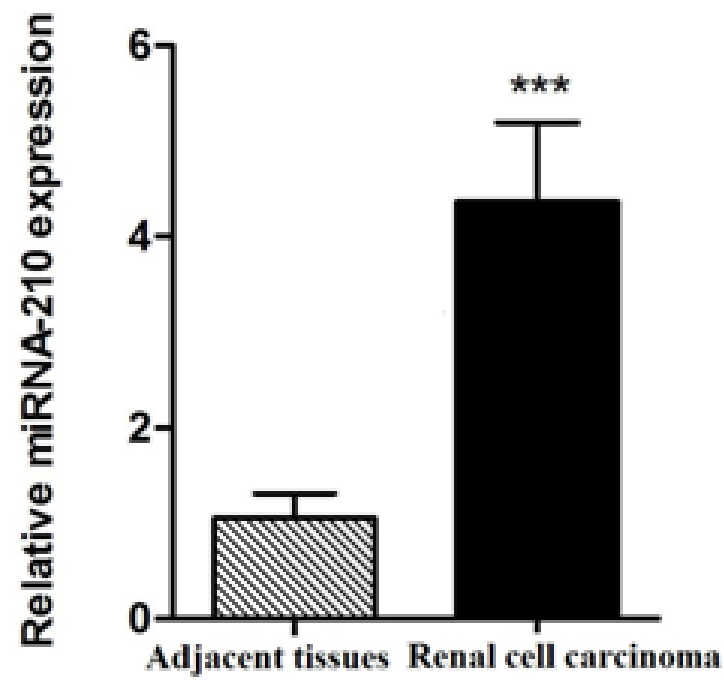

Figure 1. Expression of miR-210 in renal carcinoma and paracarcinoma tissues by real time polymerase chain reaction (compared with paracarcinoma, ${ }^{* * *} \mathrm{P}<0.001$ ).

\section{Expression of miR-210 in serum of renal carcinoma patients}

The expression of miR-210 was compared between 32 renal carcinoma patients and 32 healthy subjects. The expression of miR-210 was significantly higher $(P<0.001)$ in renal carcinoma patients than in healthy subjects (Figure 2).

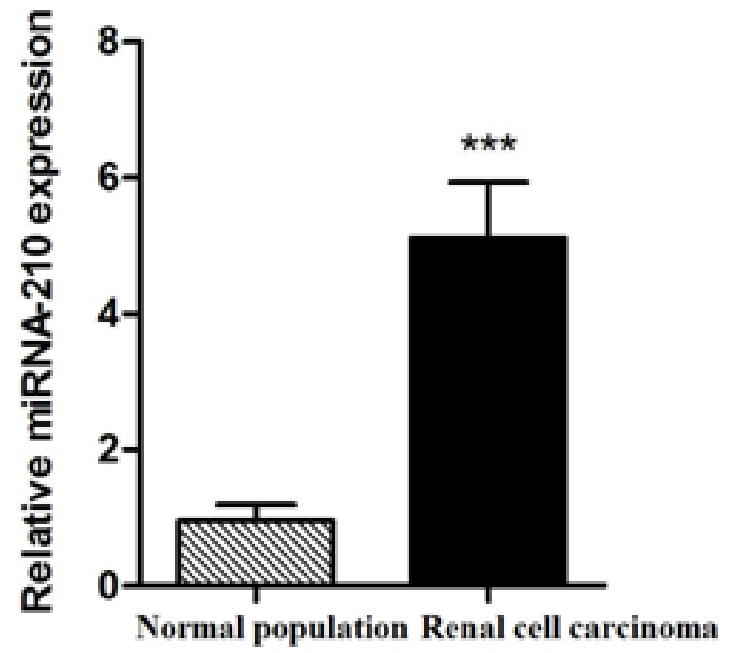

Figure 2. Expression of miR-210 in serum of normal subjects and renal carcinoma patients by real time polymerase chain reaction (compared with normal population, ${ }^{* * *} \mathrm{P}<0.001$ ). 


\section{Effects of miR-210 on renal carcinoma cell proliferation, apoptosis, and invasion}

RT-PCR quantification showed that miR-210 levels were significantly increased after ACHN cells were transfected into the control and miR-210 mimic (Figure 3A), indicating that miR210 was successfully transfected into cells. The CCK-8 proliferation experiment showed that miR210 could significantly promote the proliferation of ACHN cells (Figure 3B). Apoptosis detection by flow cytometry showed that overexpression of miR-210 significantly inhibited ACHN cell apoptosis. Early cell apoptosis and total apoptosis levels were significantly decreased compared with the control group $(P<0.05)$ (Figure $3 C$ ). The transwell invasion experiment results showed that the number of cells below the transwell chamber was significantly higher $(P<0.05)$ in ACHN cells transfected with miR-210 than in the control group (Figure 3D). The above results suggested that miR-210 could promote the proliferation and invasion of renal carcinoma cells and inhibit the apoptosis of renal carcinoma cells.

A

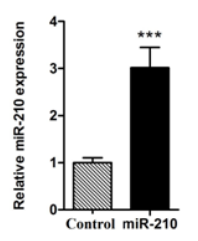

$\mathrm{C}$

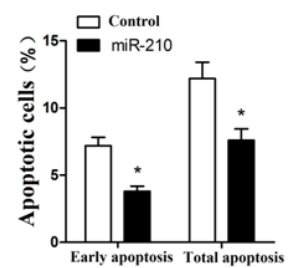

B

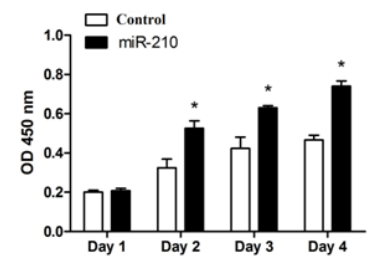

D

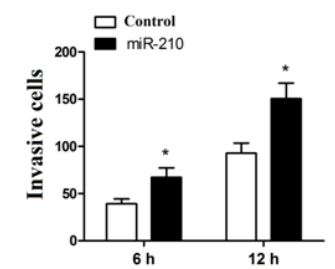

Figure 3. Effects of miR-210 on renal carcinoma cell proliferation, apoptosis, and invasion (compared with control group, $\left.{ }^{*} \mathrm{P}<0.05 ;{ }^{* * *} \mathrm{P}<0.001\right)$. A. Relative miR-210 expression; B. CCK-8 proliferation experiment results; C. apoptosis levels; D. invasion levels.

In addition, the two-factor repeated measure analysis of the OD value $(450 \mathrm{~nm})$ was conducted. The results showed that $F$ and $P$ were respectively: intergroup: 89.073, 0.000; time-point: 841.855, 0.000; group *time-point: 102.142, 0.000. These values were significant, suggesting that the OD values and variation trends were different between the two groups.

To further verify the effects of miR-210 on ACHN cell proliferation, apoptosis, and invasion, the functional loss experiment (low miR-210 expression) was conducted again. After ACHN cells were transfected with the miR-210 inhibitor, the level of miR-210 was significantly decreased (Figure 4A), suggesting that the inhibition was successful. The CCK-8 proliferation experiment showed that the proliferation of renal carcinoma cells was significantly decreased after inhibiting miR-210 compared to the control group (Figure 4B). In addition, the early apoptosis and total apoptosis levels were increased significantly $(P<0.05)$ (Figure $4 C)$. The transwell invasion assay showed that the invasion level of renal carcinoma cells was significantly decreased $(P<0.05)$ (Figure 4D). The results of the functional loss experiment indicate that miR-210 is involved in the regulation of renal carcinoma cell proliferation, apoptosis, and invasion. 
A

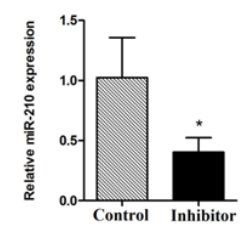

$\mathrm{C}$

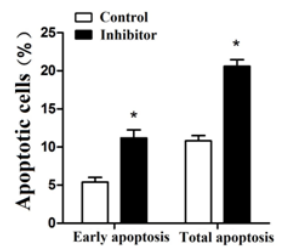

B

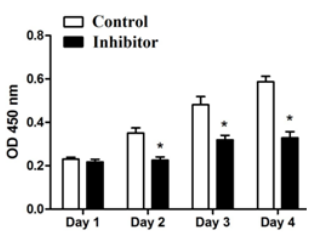

$\mathrm{D}$

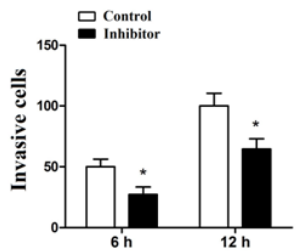

Figure 4. Effects of low expression miR-210 on renal carcinoma cell proliferation, apoptosis, and invasion (compared with control group, ${ }^{*} \mathrm{P}<0.05$ ). A. Relative miR-210 expression; B. CCK-8 proliferation experiment results; C. apoptosis levels; $\mathbf{D}$. invasion levels.

The two-factor repeated measure analysis of OD $(450 \mathrm{~nm})$ values was also conducted. The results showed that $F$ and $P$ were respectively: intergroup: 75.128, 0.000; time-point: 484.290, 0.000 ; group* time-point: $110.268,0.000$. These values were all significant, suggesting that the OD values of two groups were different. The variation trends were different between two groups with the time change.

\section{DISCUSSION}

Renal cell carcinoma is a malignant tumor in the urinary system, ranking second only to prostate and bladder cancers. The mortality rate can reach $40 \%$. RCC can be divided into different histological types according to genetic and biological differences. The clear cell carcinoma of kidney (CCC) accounts for about $70 \%$ of RCC (Rathmell et al., 2005). Nearly $30 \%$ of RCC patients will occur to important organs including bone, lung, and brain metastasis. Thus, it is critical to understand the molecular mechanism of RCC to provide the basis for effective treatment.

miRNAs are single stranded small noncoding RNAs that can inhibit the translation of a protein or degrade mRNA to downregulate the expression of target genes by binding sequences in the 3' UTR. One miRNA might act on different target genes and participate in different biological processes. miRNAs are predicted to regulate nearly $50 \%$ of the genes in mammals (Bartel, 2004). The production of miRNAs is regulated by different factors. For example, many miRNAs are highly expressed in tumor tissues. miR-210 is also highly expressed in hypoxic conditions, so miR-210 is considered to be an miRNA induced by hypoxia (Kulshreshtha et al., 2007). It was reported that miR-210 was highly expressed in CCC by promoting HIF. The quantification of miR-210 in renal carcinoma and corresponding paracarcinoma tissues confirmed the high expression of miR-210 in renal carcinoma tissue. In addition, the expression of miR-210 in serum of RCC patients was higher than that in healthy subjects, suggesting that the high expression of miR-210 in carcinoma tissue and serum of renal carcinoma patients is closely related to the occurrence and development of renal carcinoma.

In order to explore the role of miR-210 in the occurrence and development of renal carcinoma, the effects of miR-210 overexpression and inhibition on renal carcinoma cell proliferation, 
apoptosis, and invasion were investigated. The results showed that overexpression of miR-210 could promote the proliferation and invasion of renal carcinoma cells, as well as inhibit apoptosis of renal carcinoma cells. After miR-210 expression in renal carcinoma cells was inhibited, proliferation and invasion of renal carcinoma cells were inhibited and apoptosis was significantly increased. These results suggested that miR-210 is involved in the pathopoiesis of renal carcinoma through promoting cell proliferation, invasion, and inhibiting apoptosis.

Many literature sources report that miR-210 could act on different target genes, such as to regulate cell cycle E2F3, cell proliferation, differentiation, and apoptosis (Biswas et al., 2010). In addition, miR-210 acted on the proliferation processes of fibroblast growth factor receptor protein 1 and homeobox A1 (Bartel, 2004; Rathmell et al., 2005; Kulshreshtha et al., 2007; Huang et al., 2009; Biswas et al., 2010; Tsuchiya et al., 2011; Cicchillitti et al., 2012; Alaiti et al., 2012; Samaan et al., 2015). A study on endothelial cells showed that miR-210 also acted on Ephrin-A3 to promote microtubule formation and migration of endothelial cells (Fasanaro et al., 2008). In hepatocellular carcinoma, miR-210 was shown to act on vacuole membrane protein 1 to promote the migration and invasion of hepatocellular carcinoma cells induced by hypoxia (Ying et al., 2011). Therefore, our study showed that miR-210 might regulate the proliferation, apoptosis, and invasion of renal carcinoma cells by acting on the above genes that are involved in the occurrence and development of renal carcinoma.

In conclusion, miR-210 was highly expressed in carcinoma tissue and serum of renal carcinoma patients. The high expression of miR-210 was involved in renal carcinoma cell proliferation, apoptosis, and invasion. These results suggest that miR-210 plays an important role in the occurrence and development of renal carcinoma, and might have some guidance for clinical individualized treatment and prognosis of renal carcinoma patients.

\section{Conflicts of interest}

The authors declare no conflict of interest.

\section{REFERENCES}

Alaiti MA, Ishikawa M, Masuda H, Simon DI, et al. (2012). Up-regulation of miR-210 by vascular endothelial growth factor in ex vivo expanded CD34+ cells enhances cell-mediated angiogenesis. J. Cell. Mol. Med. 16: 2413-2421. http://dx.doi. org/10.1111/j.1582-4934.2012.01557.x

Bartel DP (2004). MicroRNAs: genomics, biogenesis, mechanism, and function. Cell 116: 281-297. http://dx.doi.org/10.1016/ $\underline{\text { S0092-8674(04)00045-5 }}$

Biswas S, Roy S, Banerjee J, Hussain SR, et al. (2010). Hypoxia inducible microRNA 210 attenuates keratinocyte proliferation and impairs closure in a murine model of ischemic wounds. Proc. Natl. Acad. Sci. USA 107: 6976-6981. http://dx.doi. org/10.1073/pnas.1001653107

Cicchillitti L, Di Stefano V, Isaia E, Crimaldi L, et al. (2012). Hypoxia-inducible factor 1- $\alpha$ induces miR-210 in normoxic differentiating myoblasts. J. Biol. Chem. 287: 44761-44771. http://dx.doi.org/10.1074/jbc.M112.421255

Cohen HT and McGovern FJ (2005). Renal-cell carcinoma. N. Engl. J. Med. 353: 2477-2490. http://dx.doi.org/10.1056/ NEJMra043172

Doh LS, Amato RJ, Paulino AC and Teh BS (2006). Radiation therapy in the management of brain metastases from renal cell carcinoma. Oncology (Huntingt.) 20: 603-613, discussion 613, 616, 619-620 passsim.

Drucker BJ (2005). Renal cell carcinoma: current status and future prospects. Cancer Treat. Rev. 31: 536-545. http://dx.doi. org/10.1016/j.ctrv.2005.07.009

Du T and Zamore PD (2005). microPrimer: the biogenesis and function of microRNA. Development 132: 4645-4652. http:// dx.doi.org/10.1242/dev.02070

Fasanaro P, D'Alessandra Y, Di Stefano V, Melchionna R, et al. (2008). MicroRNA-210 modulates endothelial cell response to hypoxia and inhibits the receptor tyrosine kinase ligand Ephrin-A3. J. Biol. Chem. 283: 15878-15883. http://dx.doi. org/10.1074/jbc.M800731200 
Giannakakis A, Sandaltzopoulos R, Greshock J, Liang S, et al. (2008). miR-210 links hypoxia with cell cycle regulation and is deleted in human epithelial ovarian cancer. Cancer Biol. Ther. 7: 255-264. http://dx.doi.org/10.4161/cbt.7.2.5297

Huang X, Ding L, Bennewith KL, Tong RT, et al. (2009). Hypoxia-inducible mir-210 regulates normoxic gene expression involved in tumor initiation. Mol. Cell 35: 856-867. http://dx.doi.org/10.1016/j.molcel.2009.09.006

Kim VN (2005). MicroRNA biogenesis: coordinated cropping and dicing. Nat. Rev. Mol. Cell Biol. 6: 376-385. http://dx.doi. org/10.1038/nrm1644

Kulshreshtha R, Ferracin M, Wojcik SE, Garzon R, et al. (2007). A microRNA signature of hypoxia. Mol. Cell. Biol. 27: 18591867. http://dx.doi.org/10.1128/MCB.01395-06

McCormick RI, Blick C, Ragoussis J, Schoedel J, et al. (2013). miR-210 is a target of hypoxia-inducible factors 1 and 2 in renal cancer, regulates ISCU and correlates with good prognosis. Br. J. Cancer 108: 1133-1142. http://dx.doi.org/10.1038/ bjc. 2013.56

Nakada C, Tsukamoto Y, Matsuura K, Nguyen TL, et al. (2011). Overexpression of miR-210, a downstream target of HIF1a, causes centrosome amplification in renal carcinoma cells. J. Pathol. 224: 280-288. http://dx.doi.org/10.1002/path.2860

Rathmell WK, Wright TM and Rini BI (2005). Molecularly targeted therapy in renal cell carcinoma. Expert Rev. Anticancer Ther. 5: 1031-1040. http://dx.doi.org/10.1586/14737140.5.6.1031

Samaan S, Khella HW, Girgis A, Scorilas A, et al. (2015). miR-210 is a prognostic marker in clear cell renal cell carcinoma. J. Mol. Diagn. 17: 136-144. http://dx.doi.org/10.1016/j.jmoldx.2014.10.005

Takikawa T, Masamune A, Hamada S, Nakano E, et al. (2013). miR-210 regulates the interaction between pancreatic cancer cells and stellate cells. Biochem. Biophys. Res. Commun. 437: 433-439. http://dx.doi.org/10.1016/j.bbrc.2013.06.097

Tsuchiya S, Fujiwara T, Sato F, Shimada Y, et al. (2011). MicroRNA-210 regulates cancer cell proliferation through targeting fibroblast growth factor receptor-like 1 (FGFRL1). J. Biol. Chem. 286: 420-428. http://dx.doi.org/10.1074/jbc.M110.170852

Ying Q, Liang L, Guo W, Zha R, et al. (2011). Hypoxia-inducible microRNA-210 augments the metastatic potential of tumor cells by targeting vacuole membrane protein 1 in hepatocellular carcinoma. Hepatology 54: 2064-2075. http://dx.doi. org/10.1002/hep.24614

Zhang Z, Sun H, Dai H, Walsh RM, et al. (2009). MicroRNA miR-210 modulates cellular response to hypoxia through the MYC antagonist MNT. Cell Cycle 8: 2756-2768. http://dx.doi.org/10.4161/cc.8.17.9387 